Contributions to Game Theory and Management, XIII, 388-401

\title{
Optimal Pricing Structure in a Maintenance Contract: A Game Theory Approach
}

\author{
Henrique P.Z. Santos ${ }^{1}$, Bruno N. Guedes ${ }^{2}$ and Claudio T. Cristino ${ }^{1}$ \\ 1 Federal Rural University of Pernambuco, \\ Postgraduate Program in Biometrics and Applied Statistics, \\ Rua Dom Manuel de Medeiros, s/n - Dois Irmãos, Recife - PE, 52171-900, Brazil \\ E-mail: santos .henrique624@gmail.com \\ 2 Federal University of Pernambuco, \\ Postgraduate Program in Economics, \\ Av. Prof. Moraes Rego, 1235 - Cidade Universitária, Recife - PE, 50670-901, Brazil
}

\begin{abstract}
This essay presents a novel look at Murthy and Asgharizadeh's study (Murthy \& Asgharizadeh, 1998). The authors developed a decision problem applied to maintenance outsourcing involving two decision-makers (players). If a consumer buys a product, then outsources the maintenance actions to a maintenance agent (agent) who offers two maintenance options; a maintenance contract that holds a penalty clause which is activated if the agent's time to repair is higher than a specified time, and services on-demand. The model yields equilibrium strategies based on the subgameperfect Nash equilibrium. The agent defines the optimal pricing structure for the maintenance options considering the equipment's useful life while the consumer maximizes their expected payoff by choosing one maintenance option. Our contribution to this research branches in three ways. First, once the model deals with random variables, it represents a stochastic optimization problem. We propose a different approach to estimate this penalty time by using the Monte Carlo method. The second contribution is to present a formal definition of this decision problem as a game, emphasizing the game theory's components. Finally, we reinterpret the players' equilibrium strategies.
\end{abstract}

Keywords: Game theory. Maintenance outsourcing. Simulation. Equilibrium strategies. Expected payoffs

\section{Introduction}

After-sales services play an essential role in the consumer's purchase decision, since nowadays the devices, particularly gadgets and appliances, are more complex and sophisticated. It turns out to be difficult for the consumer, the owner of the product, to carry out maintenance in-house due to lack of expertise. As a result, maintenance outsourcing has become a trend adopted by many consumers to repair their products.

Conceptually, maintenance outsourcing involves some or all maintenance actions carried out by a maintenance agent (agent) under a maintenance service contract. This document specifies the maintenance terms, incentives (or penalties) related to the product's performance for an agreed period of time. The agent in turns charges a price for such service (Murthy \& Jack, 2014).

Under a management decision, companies outsource maintenance aiming the following advantages: (i) access to high-level specialists and latest maintenance technology, (ii) better maintenance due to expertise of the maintenance agent, (iii) risk 
control, as fixed cost, the maintenance service contract removes the risk of high costs, (iv) less capital investment for the owner of the equipment, and (v) focus on core activities, managers can devote more time to other facets of the business since maintenance management demands less of their time and effort (Jackson \& Pascual, 2008).

It is relevant to consider this environment is built up by considering two decisionmakers (players) that have different perspectives concerning this service. First, the provision of maintenance implies costs, which are referred to as warranty servicing costs (Shafiee \& Chukova, 2013). They are the sum of the costs associated with the servicing of a failed item under the coverage period (Murthy \& Blischke, 2006). According to Murthy (Murthy, 2007), such costs vary from 2-10\% of the sale price of the product. Second, the agent must define the maintenance pricing complies with the repair costs and their profit. Finally, the consumer, based on the agent's maintenance price, evaluates if the maintenance price is reasonable to buy it.

The interaction between these two main parties - the agent and the consumer - can form a game theory model (Murthy et al., 2015). Each player has different goals, and decisions made for a party affect the outcomes for all the other parties. Under this context, game theory, a branch of modern applied mathematics that aims to analyze problems that involve a conflict of interests between different decisionmakers (Petrosyan \& Zenkevich, 2016).

Within the maintenance study, it is also essential to emphasize the role of uncertainty that affects decision-making among the parties involved. All products are unreliable in the sense that they may fail. A failure may be associated with manufacturing defects or due to the degradation process that is dependent on age and usage (Murthy \& Jack, 2003). Thus, understanding future costs (which are random variables) by considering both perspectives is crucial to balance the agent's profit and the consumer's maintenance cost (Rahman \& Chattopadhyay, 2015).

A seminal paper that involves all the features mentioned above was proposed by Murthy and Asgharizadeh (Murthy \& Asgharizadeh, 1998). The authors developed a quantitative model applied to maintenance outsourcing, considering the agent and the consumer's points of view.

Generally speaking, the consumer's decision regarding buying a product, a repairable good, is influenced by the post-sale service of maintenance since the consumer outsources the maintenance actions to an agent who offers two maintenance options. The model yields equilibrium strategies for all parties through the subgameperfect Nash equilibrium (Osborne, 2004). The agent defines a pricing structure considering the equipment's useful life. Alternatively, the consumer replies for each pricing structure defined by the agent.

Based on that, our contribution has three aims:

1 Apply computational statistics techniques to estimate the maintenance expected cost incurred by the agent and the consumer's expected payoff. In particular, we apply the Monte Carlo method (Taha, 2017) to perform random sampling with reliability-related performance measures to estimate their stochastic parameters.

2 Formalize this quantitative model as a sequential two-person game with perfect information. We carefully analyze the game theory elements inserted in this research.

3 Reinterpret the players' equilibrium strategies. 
The outline of the paper is as follows. In Section 2, the model is presented as a game considering all game theory components. Section 3 explains the model solution, highlighting the players' equilibrium strategies. In Section 4, a numerical example is presented. Further, this section also compares the results from our simulation with the results of the authors, underlining the computational perspective. Finally, Section 5 presents concluding remarks and provides further extensions.

\section{Model Formulation}

The model to be explained follows the steps of the systems approach proposed by Murthy and Blischake (Murthy \& Blischke, 1992). The essential elements are (i) the set of players, (ii) the set of strategies, (iii) the product's characteristics, (iv) the product's performance, (v) maintenance costs, (vi) players interaction (power configuration level), (vii) the players' equilibrium strategies, and (viii) the players' payoffs.

We follow the same structure and assumptions of the Murthy and Asgharizadeh's study (Murthy \& Asgharizadeh, 1998).

\subsection{Set of Players}

The model holds two players, a maintenance agent, and a consumer.

\subsection{Problem Description}

If the consumer purchases a product, a repairable system whose price is $P_{E}$, then it generates financial revenue $R$ per time unit when the device is working and zero if it is at the failed state during its useful life $L$. Once the consumer has no expertise to repair the product, the agent offers two maintenance options:

$O_{1}$ - Maintenance Contract. This option can be deemed as a free replacement warranty policy (Thomas \& Rao, 1999). The agent repairs the product during its useful lifetime $L$. Furthermore, there is a penalty clause that states that if the equipment is not repaired within a time $\tau$ subsequent to the failure, then the agent should be charged a fine. The penalty structure is $\alpha\left(Y_{i}-\tau\right)$ if $Y_{i}>\tau$, where $\alpha$ is the penalty cost per time unit and $Y_{i}$ is the agent's time to repair the ith failure. This penalty is seen as a refund policy to be benefit the consumer. The consumer pays $P_{M C}$ for this option.

$\mathrm{O}_{2}$ - Service on Demand. Under this option the agent charges a price $P_{S D}$ for each repair intervention. In total, the consumer pays $P_{S D} N(L)$, where $N(L)$ is the number of product failures over $L$.

\subsection{Equipment Failures and Repairs}

The mathematical model used to describe the flow of failures over time follows the characteristics of the homogeneous Poisson process (Ross, 2014). This is a stochastic point process that counts the number of events (failures) along with a time interval (Gnedenko \& Ushakov, 1995).

Let $\lambda(>0)$ be the failure rate of the product, then the expected value of the number of product failures during time interval $t$ follows the Poisson distribution whose mean is $\lambda t$. Furthermore, the average time between two consecutive failures follows a random variable exponentially distributed with mean $\lambda^{-1}$.

It is worth mentioning that as the failure rate is constant and the agent only carries out corrective maintenance (Ben-Daya et al., 2016). Thus, after a failure, the 
agent repairs the item. Under the homogeneous Poisson process, the device is not susceptible to suffer from the degradation process since the failure pattern shows no trend with time (Pulcini, 2003).

Finally, the agent's repair times are independent and identically distributed random variables that follow an exponential distribution with rate $\mu(>0)$.

\subsection{Agent's Decision Problem}

Once the coverage period is the equipment's useful life, the agent's decision problem is to define a pricing vector to the maintenance options. Let $P_{A}$ the agent's set of strategies and $\hat{p}_{A}$ a strategy from this set, where

$$
\begin{gathered}
\hat{p}_{A}=\left(P_{M C}, P_{S D}\right) \\
\hat{p}_{A} \in P_{A}
\end{gathered}
$$

\subsection{Consumer's Decision Problem}

The consumer's choice among two maintenance options is influenced by $\hat{p}_{A}$ selected by the agent, as well as by $\beta$, the coefficient of risk aversion that captures the consumer's uncertainty due to the presence of random variables related to reliability. As a result, $x\left(\hat{p}_{A}\right)$, the consumer's strategy can assume three possible values which are detailed in Table 1.

Table 1. The possible values of the consumer's strategy.

\begin{tabular}{cl}
\hline$x\left(\hat{p}_{A}\right)$ & Description \\
\hline 0 & The consumer does not purchase the product. \\
1 & The consumer purchases the product and chooses maintenance option $O_{1}$. \\
2 & The consumer purchases the product and chooses maintenance option $O_{2}$. \\
\hline
\end{tabular}

\subsection{Assumptions}

The model hols three assumptions:

A1 All elements of the structure of the game are known to all players (complete information);

A2 Once the failure-repair-failure cycle represents an homogeneous Poisson process, then we have $\mu^{-1} \ll \lambda^{-1}$, so that revenue generated by the equipment over its useful life can be approximated by $R L$;

A3 The agent's maintenance cost per failure does not change over time, corresponding to $C_{A}$.

Assumption A1 implies that the consumer knows the product's failure rate $(\lambda)$ and the agent's repair rate $(\mu)$ while the agent knows the consumer's coefficient of risk aversion $(\beta)$.

Assumption A2 states that the sum of repair times are negligible when they are compared to the product's useful life. Thus, even though the agent may incur in penalty time, this time does not affect the player's payoff. Furthermore, this assumption fits with the fact that the number of product failures is Poisson distributed with intensity $\lambda$ (Chun \& Tang, 1995).

Assumption A3 may be supported when the labor costs of handling and diagnosis dominate the warranty servicing costs (Glickman \& Berger, 1976). 


\subsection{Order of Moves}

At first, the agent defines the pricing vector for the maintenance options. Then, the consumer incorporates this information in their set of admissible control and chooses a value for $x\left(\hat{p}_{A}\right)$. This sequential structure with finite sequence of steps can be seen as a extensive-form game.

\subsection{Payoff Functions}

For each combination of strategies selected by the players, we obtain the players' payoff functions.

Consumer's expected utility. We define the consumer's payoff function as an exponential utility function that holds the coefficient of risk aversion Eq.(1)

$$
U_{C}(\gamma)=\frac{1-e^{-\beta \gamma}}{\beta}
$$

where $U_{C}$ is the consumer's utility associated with monetary gain $\gamma(\geq 0)$ due to the consumer's decision.

The advantage of this utility function is that the initial wealth is of no importance. Moreover, this function captures the attitude to risk. The risk aversion increases with $\beta$ (Rinsaka \& Sandoh, 2006). A study about the properties of this utility function can be seen in (Wood \& Khosravanian, 2015).

The consumer's monetary gain for $x\left(\hat{p}_{A}\right)=1$ is given by

$$
\gamma(1)=R L+\alpha\left[\sum_{i=0}^{N(L)} \max \left(0, Y_{i}-\tau\right)\right]-P_{M C}-P_{E} .
$$

The consumer's monetary gain for $x\left(\hat{p}_{A}\right)=2$ is given by

$$
\gamma(2)=R L-P_{S D} N(L)-P_{E}
$$

Finally, if $x\left(\hat{p}_{A}\right)=0$, the consumer's monetary gain is given by

$$
\gamma(0)=0
$$

From Eqs. (1) and (2), the consumer's expected utility when $x\left(\hat{p}_{A}\right)=1$ is:

$$
\begin{aligned}
U_{C}\left(1 ; \hat{p}_{A}\right) & =\frac{1}{\beta}\left(1-e^{-\beta\left\{R L+\alpha\left[\sum_{i=0}^{N(L)} \max \left(0, Y_{i}-\tau\right)\right]-P_{M C}-P_{E}\right\}}\right) \\
U_{C}\left(1 ; \hat{p}_{A}\right) & =\frac{1}{\beta}\left(1-e^{-\beta\left(R L-P_{M C}-P_{E}\right)} e^{-\beta \alpha\left[\sum_{i=0}^{N(L)} \max \left(0, Y_{i}-\tau\right)\right]}\right) \\
\mathrm{E}\left[U_{C}\left(1 ; \hat{p}_{A}\right)\right] & =\frac{1}{\beta}\left(1-e^{-\beta\left(R L-P_{M C}-P_{E}\right)} \mathrm{E}\left[e^{-\beta \alpha\left[\sum_{i=0}^{N(L)} \max \left(0, Y_{i}-\tau\right)\right]}\right]\right) .
\end{aligned}
$$

From Eqs. (1) and (3), the consumer's expected utility when $x\left(\hat{p}_{A}\right)=2$ is:

$$
\begin{aligned}
U_{C}\left(2 ; \hat{p}_{A}\right) & =\frac{1}{\beta}\left(1-e^{-\beta\left(R L-P_{S D}-P_{E}\right)}\right) \\
U_{C}\left(2 ; \hat{p}_{A}\right) & =\frac{1}{\beta}\left(1-e^{-\beta\left(R L-P_{E}\right)} e^{\beta P_{S D} N(L)}\right) \\
\mathrm{E}\left[U_{C}\left(2 ; \hat{p}_{A}\right)\right] & =\frac{1}{\beta}\left(1-e^{-\beta\left(R L-P_{E}\right)} \mathrm{E}\left[e^{\beta P_{S D} N(L)}\right]\right) .
\end{aligned}
$$


Since $N(L)$ follows the Poisson distribution with mean $\lambda L$, then $\mathrm{E}\left[e^{\beta P_{S D} N(L)}\right]$ can be seen as the moment generating function of the Poisson distribution, being rewritten as $e^{\lambda L\left(e^{\beta P_{S D}}-1\right)}$. Consequently, Eq. (6) may be manipulated to find:

$$
\begin{aligned}
& \mathrm{E}\left[U_{C}\left(2 ; \hat{p}_{A}\right)\right]=\frac{1}{\beta}\left(1-e^{-\beta\left(R L-P_{E}\right)} e^{\lambda L\left(e^{\left.\beta P_{S D}-1\right)}\right)}\right. \\
& \mathrm{E}\left[U_{C}\left(2 ; \hat{p}_{A}\right)\right]=\frac{1}{\beta}\left(1-e^{-\beta\left(R L-P_{E}\right)+\lambda L\left(e^{\left.\beta P_{S D}-1\right)}\right) .}\right.
\end{aligned}
$$

From Eqs. (1) and (4), the consumer's expected utility when $x\left(\hat{p}_{A}\right)=0$ is:

$$
\mathrm{E}\left[U_{C}\left(0 ; \hat{p}_{A}\right)\right]=0 .
$$

Agent's expected profit. The agent's payoff function is a profit function $\left(\Pi_{A}\right)$. For each value of $x\left(\hat{p}_{A}\right)$, we have the following possibilities for the expected profit.

The agent's expected profit, if the consumer purchases the product and chooses maintenance option $O_{1}$ is

$$
\mathrm{E}\left[\Pi_{A}\left(\hat{p}_{A} ; 1\right]=P_{M C}-\alpha \mathrm{E}\left[\sum_{i=0}^{N(L)} \max \left(0, Y_{i}-\tau\right)\right]-C_{A} \lambda L .\right.
$$

The agent's expected profit, if the consumer purchases the product and chooses maintenance option $\mathrm{O}_{2}$ is

$$
\mathrm{E}\left[\Pi_{A}\left(\hat{p}_{A} ; 2\right]=\left(P_{S D}-C_{A}\right) \lambda L .\right.
$$

The agent's expected profit, if the consumer does not purchase the product is

$$
\mathrm{E}\left[\Pi_{A}\left(\hat{p}_{A} ; 0\right]=0 .\right.
$$

\section{Model Solution}

This section discusses the equilibrium strategies for all players, considering a dynamic game.

At first glance, it can be seen that the decision problem presented is a nonzerosum two-person finite game with perfect information defined in two steps. Each player acts only once.

We compute the subgame perfect Nash equilibrium through backward induction (Fujiwara-Greve, 2015) to find the players' equilibrium strategies. Hence, we solve the model starting from the consumer's decision problem, and then we solve the agent's decision problem.

\subsection{Consumer's Equilibrium Strategy}

The consumer deals with a two-dimensional space whose axes are the maintenance option prices. For a given $\hat{p}_{A}$, the consumer compares their expected utility - Eqs. (5), (7), and (8) - and chooses the strategy which maximizes their expected payoff, the expected utility maximization principle (Von Neumann \& Morgenstern, 1947).

It is essential to mention that the consumer's purchase decision to each maintenance option is set up in a pricing interval whose upper limit is the consumer's reservation price (Varian, 1992), that is, the highest price that the consumer is willing to 
pay for a maintenance option. Above such price, the consumer does not choose the maintenance option. If the agent defines $\hat{p}_{A}$ below the consumer's reservation prices, then the consumer obtains consumer's surplus (Osborne \& Rubinstein, 2020).

To sum up, the consumer's equilibrium strategy, $\dot{x}\left(\hat{p}_{A}\right)$, is the best reply for each $\hat{p}_{A}$ offered by the agent ${ }^{1}$. Table 2 summarizes the consumer's equilibrium strategy, and its implications.

Table 2. Consumer's equilibrium strategy.

\begin{tabular}{ccc}
\hline$\dot{x}\left(\hat{p}_{A}\right)$ & Payoff analysis & Consumer's expected payoff \\
\hline 0 & $\mathrm{E}\left[U_{C}\left(1 ; \hat{p}_{A}\right)\right]<0$ and $\mathrm{E}\left[U_{C}\left(2 ; \hat{p}_{A}\right)\right]<0$ & 0 \\
1 & $\mathrm{E}\left[U_{C}\left(1 ; \hat{p}_{A}\right)\right]>\mathrm{E}\left[U_{C}\left(2 ; \hat{p}_{A}\right)\right]>0$ & $\mathrm{E}\left[U_{C}\left(1 ; \hat{p}_{A}\right)\right]$ \\
2 & $\mathrm{E}\left[U_{C}\left(2 ; \hat{p}_{A}\right)\right]>\mathrm{E}\left[U_{C}\left(1 ; \hat{p}_{A}\right)\right]>0$ & $\mathrm{E}\left[U_{C}\left(2 ; \hat{p}_{A}\right)\right]$ \\
\hline
\end{tabular}

The next part of this subsection explains how to compute the consumer's reservation prices for each maintenance option.

Consumer's reservation price for maintenance option $O_{1}$.

$$
\begin{array}{r}
\mathrm{E}\left[U_{C}\left(1 ; \hat{p}_{A}\right)\right]=0 \\
\frac{1}{\beta}\left(1-e^{-\beta\left(R L-P_{M C}-P_{E}\right)} \mathrm{E}\left[e^{-\beta \alpha\left[\sum_{i=0}^{N(L)} \max \left(0, Y_{i}-\tau\right)\right]}\right]\right)=0 \\
e^{-\beta\left(R L-P_{M C}-P_{E}\right)} \mathrm{E}\left[e^{-\beta \alpha\left[\sum_{i=0}^{N(L)} \max \left(0, Y_{i}-\tau\right)\right]}\right]=1 \\
-\beta\left(R L-P_{M C}-P_{E}\right)+\ln \mathrm{E}\left[e^{-\beta \alpha\left[\sum_{i=0}^{N(L)} \max \left(0, Y_{i}-\tau\right)\right]}\right]=\ln 1 \\
-\beta\left(R L-P_{M C}-P_{E}\right)=-\ln \mathrm{E}\left[e^{-\beta \alpha\left[\sum_{i=0}^{N(L)} \max \left(0, Y_{i}-\tau\right)\right]}\right] \\
R L-P_{M C}-P_{E}=\frac{\ln \mathrm{E}\left[e^{-\beta \alpha\left[\sum_{i=0}^{N(L)} \max \left(0, Y_{i}-\tau\right)\right]}\right]}{\beta} \\
\bar{P}_{M C}=R L-P_{E}-\frac{\ln \mathrm{E}\left[e^{-\beta \alpha\left[\sum_{i=0}^{N(L)} \max \left(0, Y_{i}-\tau\right)\right]}\right]}{\beta},
\end{array}
$$

where $\bar{P}_{M C}$ is the consumer's reservation price for maintenance option $O_{1}$.

\footnotetext{
${ }^{1}$ If the consumer's best reply is unique, we have the case of the Stackelberg equilibrium solution (Tamer \& Olsder, 1992).
} 
Consumer's reservation price for maintenance option $\mathrm{O}_{2}$.

$$
\begin{array}{r}
\mathrm{E}\left[U_{C}\left(2 ; \hat{p}_{A}\right)\right]=0 \\
\frac{1}{\beta}\left(1-e^{-\beta\left(R L-P_{E}\right)+\lambda L\left(e^{\beta P_{S D}}-1\right)}\right)=0 \\
e^{-\beta\left(R L-P_{E}\right)+\lambda L\left(e^{\beta P_{S D}}-1\right)}=1 \\
-\beta\left(R L-P_{E}\right)+\lambda L\left(e^{\beta P_{S D}}-1\right)=\ln 1 \\
\lambda L\left(e^{\beta P_{S D}}-1\right)=\beta\left(R L-P_{E}\right) \\
e^{\beta P_{S D}}-1=\frac{\beta\left(R L-P_{E}\right)}{\lambda L} \\
e^{\beta P_{S D}}=\frac{\beta\left(R L-P_{E}\right)}{\lambda L}+1 \\
\beta P_{S D}=\ln \left\{\frac{\beta\left(R L-P_{E}\right)}{\lambda L}+1\right\} \\
\bar{P}_{S D}=\frac{1}{\beta} \ln \left\{\frac{\beta\left(R L-P_{E}\right)}{\lambda L}+1\right\}
\end{array}
$$

where $\bar{P}_{S D}$ is the consumer's reservation price for maintenance option $\mathrm{O}_{2}$.

\subsection{Agent's Equilibrium Strategy}

The agent anticipates what the consumer will choose in the second stage and incorporates the consumer's equilibrium strategy to define their equilibrium strategy $\left(\dot{p}_{A}\right)$. The maintenance provider develops a pricing structure able to enforce the consumer the select the maintenance option that maximizes their expected profit.

Under this context, the consumer's reservation price plays an essential role in the agent's payoff. Like the consumer, the agent has a pricing range to set up the maintenance option prices. The bottom limit is the agent's cost of servicing failures under the coverage period for each maintenance option since, below these prices, the agent's payoff is negative.

Alternatively, the consumer's reservation price represents the upper for each maintenance option since, above such prices, the consumer does not purchase any maintenance option, and the agent's payoff is zero.

If the agent considers the consumer's reservation prices to determine their equilibrium strategy, we have a case of first-degree price discrimination (Varian, 1989). Consequently, the consumer's surplus is zero, and the maintenance provider captures all the benefit that the consumer receives due to the purchase of the product and the maintenance option chosen.

The agent compares which of the consumer's reservations prices $\left(\bar{P}_{M C}, \bar{P}_{S D}\right)$ provide the maximum expected profit and enforces the consumer chooses the maintenance option required following this structure:

* If maintenance option $O_{1}$ provides the maximum expected profit to the agent, we have: $\dot{p}_{A}=\left(P_{M C}=\bar{P}_{M C}, P_{S D}>\bar{P}_{S D}\right)$, and the agent's payoff is given by Eq. (9); 
* If maintenance option $O_{2}$ provides the maximum expected profit to the agent, we have: $\dot{p}_{A}=\left(P_{M C}>\bar{P}_{M C}, P_{S D}=\bar{P}_{S D}\right)$, and the agent's payoff is given by Eq. (10).

Finally, whether both maintenance options provide a negative payoff for the agent, the agent's equilibrium strategy is: $\dot{p}_{A}=\left(P_{M C}>\bar{P}_{M C}, P_{S D}>\bar{P}_{S D}\right)$.

\subsection{Simulation}

The main contribution of this research relies on applying computational statistics techniques to estimate the players' payoff under maintenance option $O_{1}$. These payoff functions possess stochastic parameters that may be estimated by the Monte Carlo method. As a result, we provide a new approach to calculate the agent's expected maintenance cost, Eq. (9), and the consumer's reservation price for the maintenance contract, Eq. (12).

In broad terms, the focus on the Monte Carlo method is based on performing random sampling in the penalty time, once it is affected by randomness since the number of product failures, as well as the agent's time to repair, are random variables. We can repeat this scenario several times to develop an empirical estimator to the mean.

Under a probability perspective, we can see the penalty time as a particular sort of the linear transformation of independent exponential random variables. When the difference between $Y_{i}$ and $\tau$ is positive, then we have such linear transformation, otherwise assume 0 (the penalty time is a non-negative continuous data).

Simulation of the agent's penalty time. The random component that affects the agent's cost under maintenance option $O_{1}$ is the penalty time. Thus, this simulation aims to estimate the mean of the penalty time incurred by the agent. Algorithm 1 shows the steps to calculate it.

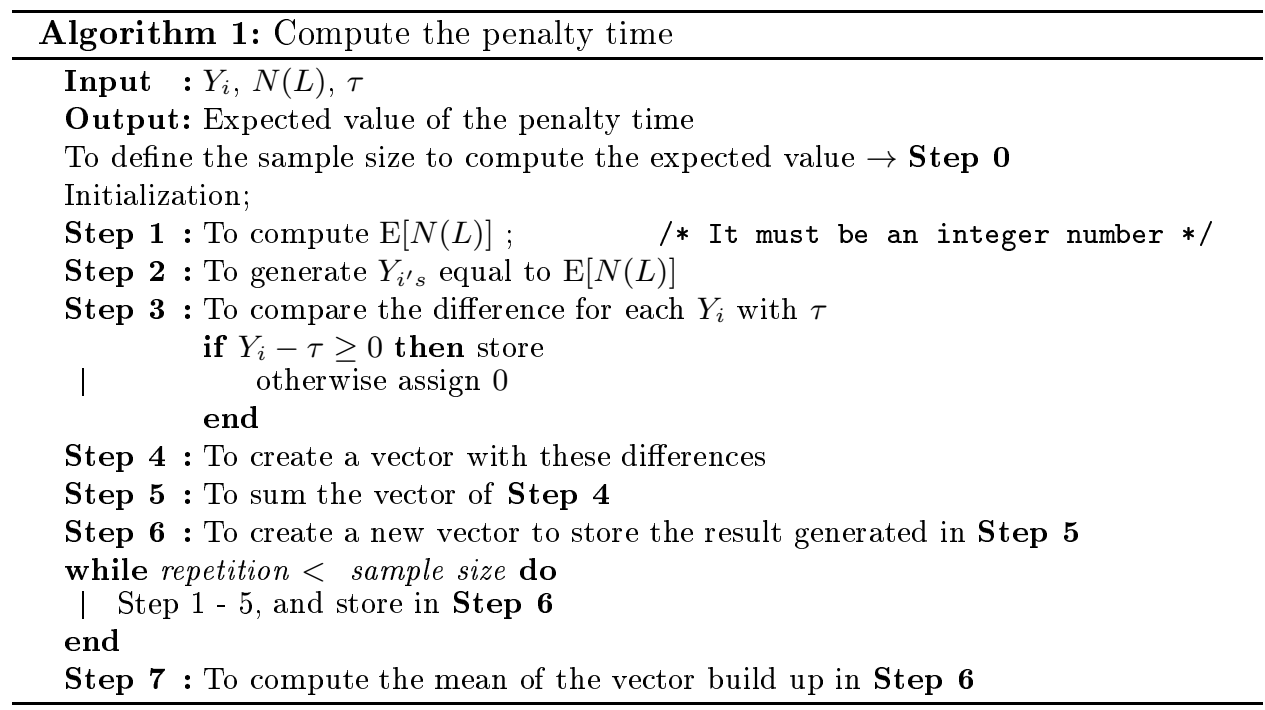

Simulation of the moment generating function of the penalty time. Based on Eq. (12), the random component present in the consumer's reservation price for 
the maintenance contract is the moment generating function of the penalty time, $\mathrm{E}\left[e^{-\beta \alpha\left[\sum_{i=0}^{N(L)} \max \left(0, Y_{i}-\tau\right)\right]}\right]$. As a result, the main target of simulation is to estimate this moment generating function.

Figure 3.3. describes the algorithm that computes the moment generating function of the penalty time.

\begin{tabular}{|c|}
\hline $\begin{array}{l}\text { Penalty time for each simulation } \\
\text { Input: } Y_{i}, N(L), \tau \\
\text { Result: Expected value of the penalty time }\end{array}$ \\
\hline $\begin{array}{l}\text { - To define the sample size to compute the expected value of the penalty time } \\
\text { - Step } 0 \text {. }\end{array}$ \\
\hline Model Execution \\
\hline $\begin{array}{l}\text { Step 1: To compute } \mathbb{E}[N(L)] \Rightarrow \text { It must be an integer number; } \\
\text { Step 2: To generate } Y_{i(s)} \text { equal to } \mathbb{E}[N(L)] \text {; }\end{array}$ \\
\hline $\begin{array}{l}\text { Step 3: To compare the difference between each } Y_{i} \text { and } \tau \text {. } \\
\quad \text { If this difference is positive then store, otherwise assign } 0 \text {. } \\
\text { Step 4: To create a vector with these differences. To sum this vector for } \\
\text { obtaining a scalar; } \\
\text { Step 5: To create a new vector to store the output generated in Step 4; } \\
\text { Loop: Repeat this procedure (Step } 1-4 \text { ) and store in Step } 5 \text { until Step 0; } \\
\text { Step 6: To compute the mean of the vector build up in Step } 5 \text {. }\end{array}$ \\
\hline
\end{tabular}

Fig. 1. Algorithm to compute the moment generating function of the penalty time.

\section{Numerical Example}

In this section, we use the same data from Murthy and Asgharizadeh's study (Murthy \& Asgharizadeh, 1998) to compare the results from our simulation with the original results found by the authors. The following nominal values for the model parameters are: $\lambda=0.0008$ (per hour), $\mu=0.02$ (per hour), $\alpha=0.06\left(10^{3} \$\right.$ per hour), $\beta=0.1, \tau=70$ (hours), $P_{E}=300\left(10^{3} \$\right), L=40,000$ (hours), $C_{A}=5$ $\left(10^{3} \$\right)$, and $R=0.015\left(10^{3} \$\right.$ per hour $)$.

Before showing the players' equilibrium strategy, we present the results of our simulation.

\subsection{Analysis of Simulation Results}

Penalty time. Table 3 resumes the expected value of the penalty time considering the number of repetitions $(\mathrm{N})$ and the standard deviation $(\mathrm{Sd})$.

After 10,000 repetitions, the expected value of the penalty time relies on an interval between 394 and 397 hours. We can assume that the real mean belongs to this time interval. Finally, the standard deviation, along with the simulations, did not present a high variability as the number of repetitions had increased. 
Table 3. Monte Carlo evaluation for the penalty time.

\begin{tabular}{ccc}
\hline $\mathrm{N}$ & Expected value & $\mathrm{Sd}$ \\
\hline 100 & 392.12 & 183.42 \\
1000 & 394.54 & 192.57 \\
5000 & 397.20 & 191.64 \\
10000 & 396.71 & 189.91 \\
20000 & 396.53 & 188.89 \\
30000 & 395.90 & 187.54 \\
40000 & 394.67 & 187.07 \\
50000 & 394.51 & 187.01 \\
\hline
\end{tabular}

Therefore, we admit that the expected time of the penalty time is 394.51 hours, the result of our last simulation.

Moment generating function of the penalty time. Table 4 resumes the value of the moment generating function of the penalty time considering the number of repetitions $(\mathrm{N})$ and the standard deviation $(\mathrm{Sd})$.

Table 4. Monte Carlo evaluation for the moment generating function of the penalty time.

\begin{tabular}{ccc}
\hline $\mathrm{N}$ & Moment generating function & $\mathrm{Sd}$ \\
\hline 100 & 0.1652 & 0.1858 \\
1000 & 0.1553 & 0.1474 \\
5000 & 0.1538 & 0.1478 \\
10000 & 0.1537 & 0.1478 \\
20000 & 0.1532 & 0.1468 \\
30000 & 0.1531 & 0.1462 \\
40000 & 0.1539 & 0.1463 \\
50000 & 0.1541 & 0.1465 \\
\hline
\end{tabular}

The results provided by Table 4 shows that the value of the moment generating function of the penalty time is almost constant, regardless of the number of simulations performed. Thus, we state the real value of the moment generating function is between 0.15 and 0.16 , a low level of variability.

To compute the consumer's reservation price for the maintenance option $O_{1}$, we assume that the value of the moment generating function is 0.1541 , due to 50,000 simulations performed.

\subsection{Equilibrium Strategies}

Reliability-related performance measures:

$\Longrightarrow \mathrm{E}[N(L)]=32$

$\Longrightarrow \mathrm{E}[$ penalty time $]=394.31$ hours

Consumer's reservation prices:

$\Longrightarrow \bar{P}_{M C}=318.6973\left(10^{3} \$\right)$ - the consumer's reservation price for the maintenance contract (Option $O_{1}$ ). Above this price, the consumer does not buy this maintenance option. 
$\Longrightarrow \bar{P}_{S D}=6.6140\left(10^{3} \$\right)$ the consumer's reservation price for the service on demand (Option $O_{2}$ ). Thus, this price is the consumer's willingness to pay for each repair intervention. Above this price, the consumer does not buy this maintenance option.

Agent's expected maintenance costs:

$\Longrightarrow$ Maintenance Option $O_{1}=183.66\left(10^{3} \$\right)$

$\Longrightarrow$ Maintenance Option $O_{2}=160\left(10^{3} \$\right)$

Agent's expected profit:

If the agent defines their equilibrium strategy considering the consumer's reservation prices, we have these two possibilities of expected profit:

$\Longrightarrow \mathrm{E}\left[\Pi_{A}\left(\hat{p}_{A} ; 1\right]=135.0387\left(10^{3} \$\right)\right.$

$\Longrightarrow \mathrm{E}\left[\Pi_{A}\left(\hat{p}_{A} ; 2\right]=51.6475\left(10^{3} \$\right)\right.$

Once maintenance option $O_{1}$ provides the higher profit than maintenance option $\mathrm{O}_{2}$, then the agent enforces the consumer to purchase the maintenance contract. Therefore, the equilibrium path is:

$$
\begin{gathered}
\dot{p}_{A}=\left(P_{M C}=\bar{P}_{M C}, P_{S D}>\bar{P}_{S D}\right) \\
x\left(\dot{p}_{A}\right)=1
\end{gathered}
$$

Finally, it is essential to consider the accuracy of the results provided by our simulation with the results found by the authors. The simulations performed were associated with the players' payoff of maintenance option $O_{1}$.

We found $\bar{P}_{M C}=318.6973\left(10^{3} \$\right)$ and $\mathrm{E}\left[\Pi_{A}\left(\hat{p}_{A} ; 1\right]=135.0387\left(10^{3} \$\right)\right.$, whereas the authors found $\bar{P}_{M C}=318.210\left(10^{3} \$\right)$ and $\mathrm{E}\left[\Pi_{A}\left(\hat{p}_{A} ; 1\right]=134.537\left(10^{3} \$\right)\right.$. We conclude our results are close to the results found analytically. Thus, our different approach to compute the players' payoff by the Monte Carlo method shows its efficiency.

\section{Conclusion}

After-sales services, such as maintenance outsourcing have become a trend in recent times due to the lack of expertise to repair complex pieces of equipment in house and to the possibility of letting managers to focus on the companies core business, among other factors. Thus, it is essential to analyze the negotiation process of maintenance service contracts and provide relevant insights to support decision makers.

In this paper a two-person game considering a consumer and an agent was proposed to analyze the negotiation process of a maintenance service contract of a perfectly repairable product. While the consumer decides whether to buy the equipment or not and to sign a maintenance service contract or pay for repair interventions on demand in order to maximize their expected utility, the agent determines what prices to charge for the contract and for each maintenance intervention in order to maximize their expected profit. Under the model's assumptions, the prices set by the agent will determine the consumer's strategy, as the first hold all the bargain power involved in the negotiation process.

We can extend this research by adding a new player (the manufacturer) or a different framework on how the decision-makers set up their strategies. 
Acknowlegments. The authors express their gratitude to Fabio Jaques da Silveira and Francisco Cribari for useful discussions on the subjects.

\section{References}

Ben-Daya, M., Kumar, U., Murthy, D. N. P. (2016). Introduction to maintenance engineering: modelling, optimization and management. John Wiley \& Sons, 1, 684.

Chun, Y.H., and Tang, K. (1995). Determining the optimal warranty price based on the producer's and customers' risk preferences. European Journal of Operational Research, $\mathbf{8 5}(\mathbf{1}), 97-110$.

Fujiwara-Greve, T. (2015). Non-cooperative game theory. Springer Japan, 1, 260.

Glickman, T. S., and Berger, P.D. (1976). Optimal Price and Protection Period Decisions for a Product Under Warranty. Management Science, 22(12), 1381-1390.

Gnedenko, B., Ushakov, I. A. (1995). Probabilistic reliability engineering. John Wiley \& Sons, 1, 663 .

Jackson, C., and Pascual, R. (2008). Optimal maintenance service contract negotiation with aging equipment. European Journal of Operational Research, 189(2), 387-398.

Murthy, D. N. P. (2007). Product reliability and warranty: an overview and future research. Production, 17(3), 426-434.

Murthy, D. N. P., Asgharizadeh, E. (1998). A stochastic model for service contract. International Journal of Reliability, Quality and Safety Engineering, 5(01), 29-45.

Murthy, D. N.P., and Blischke, W.R. (1992). Product warranty management - II: An integrated framework for study. European Journal of Operational Research, 62(3), 261-281.

Murthy, D. N.P., and Blischke, W.R. (2006). Warranty management and product manufacture. Springer Science \& Business, 1, 324.

Murthy, D. N. P., Karim, M.R., \& Ahmadi, A. (2015). Data management in maintenance outsourcing. Reliability Engineering \& System Safety, 142, 100-110.

Murthy, D. N. P., and Jack, N. (2003). Warranty and Maintenance, a chapter in Handbook of Reliability Engineering, Hoang Pham, editor. Springer-Verlag London, 1, 305-316.

Murthy, D. N.P., and Jack, N. (2014). Extended warranties, maintenance service and lease contracts: modeling and analysis for decision-making. Springer Science \& Business, 1, 399.

Osborne, M. J. (2004). An introduction to game theory . New York: Oxford university press, 3, 560 .

Osborne, M. J., Rubinstein, A. (2020). Models in Microeconomic Theory. OpenBook Publishers, 1, 362.

Petrosyan, L., and Zenkevich, N. (2016). Game Theory. World Scientific Publishing Company, 2,564 .

Pulcini, G. (2003). Mechanical Reliability and Maintenance Models, a chapter in Handbook of Reliability Engineering, Hoang Pham, editor. Springer-Verlag London, 1, 317-348.

Rahman, A., and Chattopadhyay, G. (2015). Long term warranty and after sales service: Concept, Policies and Cost Models. Springer International Publishing, 1, 113.

Rinsaka, K., and Sandoh, H. (2006). A stochastic model on an additional warranty service contract. Computers \& Mathematics with Applications, 51(2), 179-188.

Ross, S. (2014). Introduction to Probability Models. Academic Press, 11, 784.

Shafiee, M., and Chukova, S. (2013). Maintenance models in warranty: A literature review. European Journal of Operational Research, 229(3), 561-572.

Taha, H. A. (2017). Operations Research An Introduction. Pearson Education Limited, 10, 848.

Tamer, B., Olsder, G. J. (1999). Dynamic noncooperative game theory. Siam, 23, 563.

Thomas, M. U., and Rao, S. S. (1999). Warranty economic decision models: A summary and some suggested directions for future research. Operations Research, 47(6), 807-820. 
Varian, H. R. (1989). "Price discrimination." Handbook of industrial organization. North Holland, 1, 597-654.

Varian, H. R. (1992). Microeconomic analysis . W. W. Norton \& Company, 3, 563.

Von Neumann, J., Morgenstern, O. (1953). Theory of games and economic behavior. Princeton university press, 3,641 .

Wood, D.A., and Khosravanian, R. (2015). Exponential utility functions aid upstream decision making. Journal of Natural Gas Science and Engineering, 27, 1482-1494. 